\title{
Balancing Retrospection and Visions: The Cytogenetics Group of the Society of Plant Breeding (GPZ) Came Together in Dresden
}

\author{
Tony Heitkam \\ Faculty of Biology, Technical University Dresden, Dresden, Germany
}

From September 26th to 27th, 2019, the Cytogenetics group of the Society of Plant Breeding (GPZ) met in a relaxed atmosphere near Dresden (Germany). This year's meeting focused on "Chromosome Biology, Genome Evolution, and Modern Cytogenetics in the Context of Plant Breeding" and was attended by 41 members from academia and industry (Fig. 1).

\section{Balancing Retrospection and Visions for the Future of Cytogenetics}

Although the first reports of mitotic cell divisions were already published over a century ago [Schneider, 1873], cytogenetics continuously offers new insights into the biology and the evolution of chromosomes and genomes. Molecular approaches introduced in the 1980s to 1990s have thoroughly rebuilt the field and made it possible to support plant breeding with unmet precision, e.g., by the generation of karyotypes or the dissection of the species' contributions to introgressions [Jiang and Gill, 2006]. Now that germplasm collections have arrived in the genomics age [Milner et al., 2019] and wild crop relatives can be domesticated by a few gene edits [Zsögön et al., 2018], also the cytogenetics field is being re-positioned again. To be able to successfully integrate big data, next-/ third-generation sequencing, oligo- and CRISPR-FISH, the cytogenetics community needs both (1) an understanding of the unresolved research questions still pushing the field, and (2) good grasps on the strengths and limitations of the modern methods. We positioned the Cytogenetics group meeting at this intersection and wanted to have a small-scale meeting, during which interaction and conversation would be easy and rewarding. Therefore, we decided on the beautiful park setting on the outskirts of Dresden, left room for discussions and strolls through the gardens, as well as organized two joint meals and a guided city tour.

The scope and also the venue of the GPZ Cytogenetics meeting has been chosen by Prof. Dr. Thomas Schmidt, former group leader of TU Dresden's research group "Plant Cell and Molecular Biology," and initiator of the meeting. In the light of his very recent, tragic, and absolutely unpredictable death, the meeting was dedicated to his memory.

That we met the balance between retrospection and the future can - to no small part - be attributed to Pat Heslop-Harrison (University of Leicester, UK, and South China Botanical Garden, Guangzhou, China) and his thoughtful keynote. He initially submitted an abstract entitled "Genomes and Crops: Domestication and Now Superdomestication," starting with: "It has never been a more exciting time to work on genome evolution, chromosomes, and crops [...]". As former mentor and long-

\section{KARGER}

(c) 2020 S. Karger AG, Basel

E-Mail karger@karger.com

www.karger.com/cgr
Tony Heitkam

Faculty of Biology, Technical University Dresden

Zellescher Weg 20b

DE-01062 Dresden (Germany)

E-Mail tony.heitkam@tu-dresden.de 


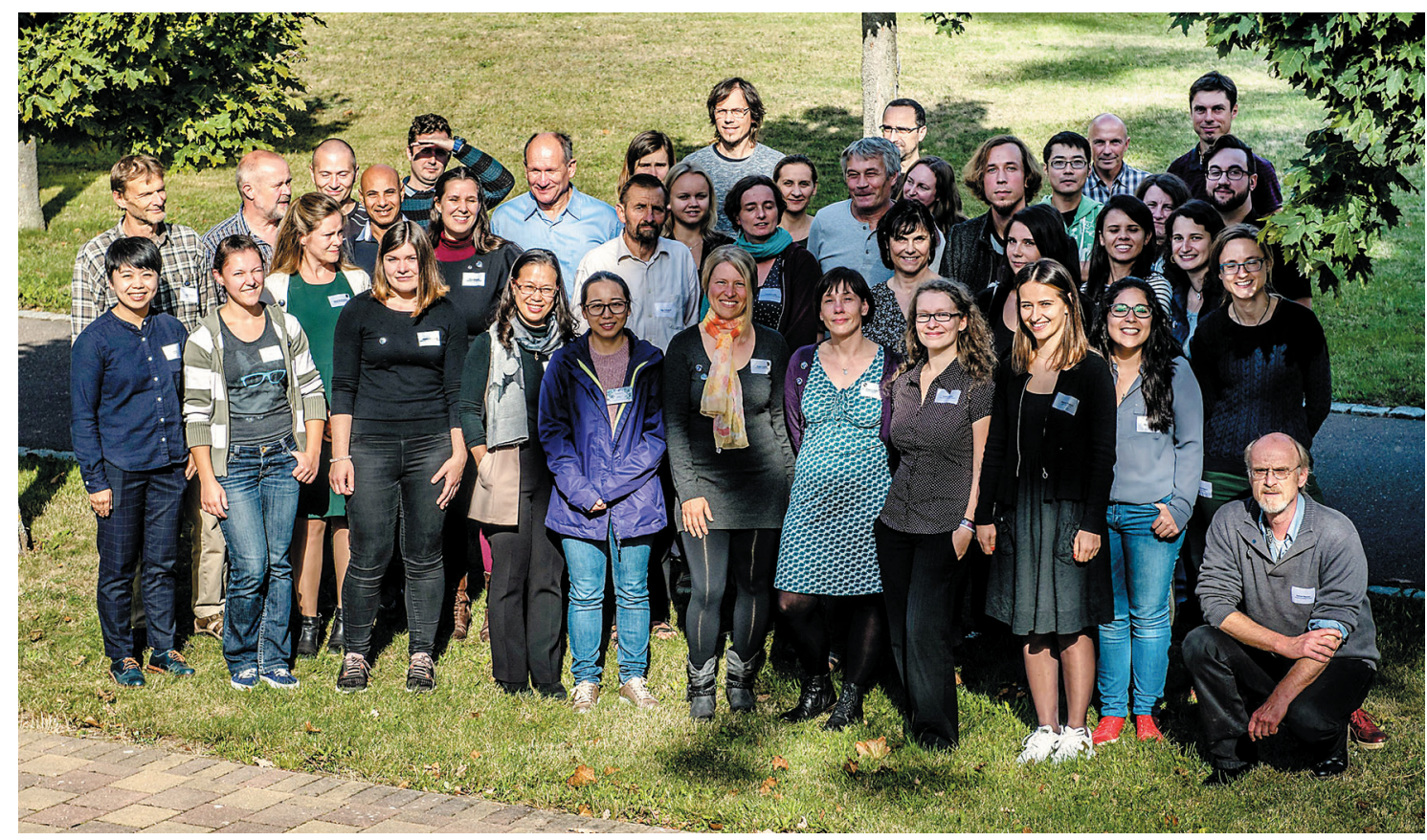

Fig. 1. Science in a relaxed environment. The GPZ Cytogenetics meeting near Dresden assembled 41 attendants. Photograph taken by Gerhard Menzel.

standing close friend to Thomas Schmidt, he rewrote his lecture to pay tribute to Thomas' scientific achievements. Pat found the right words to lead us through Thomas' life in biology, starting from his doctorate and postdoc years in Halle, the time in Norwich and Kiel, and the science coming from his lab at TU Dresden.

\section{Integration of Cytogenetics with *omics Technologies}

How *omics technologies may influence and expand the scope of cytogenetics was the topic of the first session, chaired by Andreas Houben (IPK Gatersleben, Germany). We jumped in with computer scientist Amanda Souza Câmara (IPK Gatersleben, Germany) introducing us to polymer simulations and how they can help to understand the folded structure of mitotic chromosomes. Using fluorescent in situ hybridization (FISH) with oligonucleotide probes (oligo-FISH) spanning a defined length along barley (Hordeum vulgare) chromosomes, she showed how in situ methods may help to support her simulations. Combining both analyses, she argued that the mitotic chromosome may be formed by nested loops arranged side by side in a dynamical helical scaffold.
An update on wheat (Triticum aestivum) genomics was provided by Hana Šimková (Institute of Experimental Botany of the Academy of Sciences of the Czech Republic). She informed about the progress of single-chromosome optical mapping, a technique already established in the group [Staňková et al., 2016]. To provide a realistic guide for wheat genome assembly, DNA molecules with lengths between $150 \mathrm{~kb}$ and $1 \mathrm{Mb}$ were imaged using the Bionano Genomics technology. As an example for a practical application, she demonstrated how optical mapping has helped to pin down a locus conferring resistance against Russian wheat aphids [Tulpová et al., 2019].

Next, Phoung Hoang and Ingo Schubert (IPK Gatersleben, Germany) described how integration of thirdgeneration genome sequencing and comparative multicolor FISH helped to produce the most current duckweed (Spirodela sp.) genome maps [Hoang et al., 2019]. They laid out how map discrepancies were resolved and how these results provided a more complete picture of karyotype evolution in duckweeds.

The progress on the integration of genetic and physical maps of Phalaenopsis orchid was presented by Yi-Tzu Kuo (National Taiwan University, Taiwan). She explained that for the generation of a molecular cytogenetic map, 22 linkage groups were hybridized onto the or- 
chid's 19 pachytene chromosomes. The resulting map showed high consistency with all linkage group-specific DNA markers. This high-quality genome sequence allowed for example to trace the duplication events in the evolutionary history of the chalcone synthase gene family [Kuo et al., 2019].

Vildana Suljevic (University of Vienna, Austria) and André Marques (Max Planck Institute for Plant Breeding Research, Germany) explained how they used low-coverage sequencing to determine the repeat composition of their genomes of interest: Vildana Suljevic first introduced us to genomes of the highly pungent chili peppers (Capsicum sp.) and showed that some of the very abundant satellite DNA families presumably derived from spacer regions of functional rDNAs. Similar to the peppers, the white lupin (Lupinus albus) genome is also occupied by a high amount of satellite DNAs. André Marques showed that most of its satellite DNA families are associated with CENH3-immunoprecipitated chromatin. Nevertheless, it has been possible to develop chromosomal landmark probes for the identification of most chromosome pairs.

\section{Meiosis, Gamete Formation, and Polyploidy}

The second session was chaired by Christiane Ritz (Senckenberg Museum Görlitz, Germany) and targeted the development of polyploids, gamete formation, and meiosis. Anna Nowicka (Institute of Experimental Botany of the Academy of Sciences of the Czech Republic) started the session and focused on endoreduplication processes in the developing barley seed. She and her project group studied the nuclear ploidy of the embryo, endosperm, and pericarp during seed development from pollination until full maturity. They found that endoreduplication is an essential process during grain development mainly occurring in the endosperm.

An intriguing plant model for the investigation of polyploidy was presented by Aleš Kovařík (Czech Academy of Science, Institute of Biophysics, Czech Republic). The dogroses (members of the genus Rosa, section Caninae) with ploidies of 4,5 , and 6 are marked by a meiosis with asymmetric distribution of genetic material in the gametes [Herklotz et al., 2018]. To understand the genetic origin of the unusual pentaploid dogrose, 5 dogrose species were comparatively sequenced in low coverage. Characterization of the repeat landscapes gave rise to a set of tandem repeat probes, serving as cytogenetic landmarks for comparative karyotyping. This led the project group to hypothesize that pentaploid dogroses originated by independent and reciprocal hybridization events.

Tony Heitkam (TU Dresden, Germany) presented how multi-color FISH helped to trace the origin of saffron crocus (Crocus sativus) [Schmidt et al., 2019]. She explained how the project group used whole-genome sequencing reads of low coverage to identify tandem repeats, which served as landmark probes for saffron karyotyping. Comparative FISH uncovered an autotriploidy of different cytotypes from the wild Crocus cartwrightianus. Corroborating this, Frank Blattner's group at IPK Gatersleben used genotyping by sequencing and independently arrived at the same conclusion [Nemati et al., 2019].

\section{Special Chromosome Types}

Pat Heslop-Harrison chaired the third session on special chromosome types. Andreas Houben started by giving an overview why we should pay attention to holocentric chromosomes. As holocentricity evolved independently at least 13 times (4 times in plants), he dived into the question whether holocentromeres resembled each other across the plant kingdom. Focusing on Poales models, he presented distinct holocentromere types, differing in sequence organization and dynamics.

Bimodal karyotypes combine chromosomes which are either very small or very large. Mariana Báez (Federal University of Pernambuco, Recife, Brazil, and IPK Gatersleben, Germany) introduced Eleutherine bulbosa with a very large chromosome pair complementing the 5 much smaller pairs. She posed the question whether repetitive DNA fractions vary between both chromosome sets. Indeed, retrotransposons differed in their distribution, pointing to a different organization of the bimodal subgenomes, which probably evolved by repeat accumulation on the large chromosome pair [Báez et al., 2019].

Jörg Fuchs (IPK Gatersleben, Germany) addressed why dispensable B chromosomes not only survive, but are even preferentially inherited. He used flow cytometry to sort pollen nuclei according to their vegetative or generative origin. At the same time, the number of B chromosomes was quantified [Wu et al., 2019]. This allowed to measure an accumulation of B chromosomes in generative nuclei during the first pollen mitosis and represents an accurate estimate of the strength of the $\mathrm{B}$ chromosome drive in Aegilops speltoides. 


\section{Genome Stability and Nucleus Architecture}

Chaired by Ingo Schubert, the fourth session targeted the stability of the genome and the architecture of the nucleus. Kateřina Perničková, David Kopecký, and Mahmoud Said (Institute of Experimental Botany of the Academy of Sciences of the Czech Republic) reported on the processes following alien introgressions. Kateřina Perničková asked why introgressed chromosomes were often eliminated during meiosis [Perničková et al., 2019]. Using 3D-FISH and confocal microscopy, she found that abnormal positioning of alien chromosomes may not only occur during meiosis but also in somatic nuclei. When observing alien rye (Secale cereale) chromosomes in a wheat background, the project group frequently detected incorrectly positioned rye telomeres. This abnormal telomere arrangement may reduce the rye chromosomes' potential to reach the meiotic bouquet, and thus may lead to their elimination.

On broader terms, David Kopecký divided hybrids with introgressed material into 2 types based on their meiotic behavior: in meiosis, chromosomes of type I hybrids pair exclusively between homologs, whereas type II hybrids also allow homoeologous chromosome pairing. The observed meiotic behavior may indicate the evolutionary trajectory of the hybrid, e.g., the degree of genome dominance or a potential elimination of chromosomes [Kopecký et al., 2019].

Then, Mahmoud Said argued for the importance of synteny between homoeologous loci in interspecific hybrids. He focused on 2 Aegilops species important for wheat improvement and localized 44 single-gene probes from wheat along the Aegilops chromosomes. The detected structural rearrangements provide insights into the genome evolution of grasses and may support Aegilops use in wheat breeding.

\section{New Tools, Perspectives, and Applications}

Veit Schubert (IPK Gatersleben, Germany) chaired the final session covering a range of new tools and methods advancing the cytogenetics field. Takayoshi Ishii (Tottori University, Japan) presented a CRISPR/Cas9based approach to allow visualization of defined genomic sequences in fixed nuclei. The RNA-guided endonuclease in situ labeling (RGEN-ISL) method does not require denaturation steps and hence better preserves the chromatin structure compared to conventional FISH [Ishii et al., 2019; Nemeckova et al., 2019].
"Can you magnify nuclei prior to microscopy?," asked Ivona Kubalova (IPK Gatersleben, Germany). She presented how chromatin can be physically expanded with a polyelectrolyte hydrogel, which causes the sample to swell - a new method that she transferred from mammalian [Wassie et al., 2019] to plant tissue. Wide-field microscopy showed that the treated barley nuclei retained their general structure, whereas super-resolution microscopy revealed a loss of the chromatin ultrastructure. Nevertheless, the chromatin position of expanded nuclei was maintained, as for example shown for the iconic Rabl configuration.

Updates on oligo-FISH in banana (Musa acuminata) were presented by Eva Hribova (Institute of Experimental Botany of the Academy of Sciences of the Czech Republic). She showed how chromosome painting probes were designed from the reference genome sequence and how their use may shed light on chromosomal rearrangements in banana species [Šimoníková et al., 2019].

\section{A Look at the Posters}

The meeting hosted 11 posters, which were vividly discussed. Alžběta Němečková (Institute of Experimental Botany of the Academy of Sciences of the Czech Republic) asked whether chromatin arrangements in interphase nuclei correlate with genome size. Using the root tip meristem of 7 grasses, she found that interphase nuclei of species with relatively small genomes (Brachypodium, rice and maize) contained dense chromatin in few, small regions. In contrast, interphase nuclei of species with large genomes (barley, wheat, rye, and oat) had many and more dispersed loci of compacted chromatin.

In 11 duckweeds, Phoung Hoang and Ingo Schubert investigated the inverse relationship between genome size and leaf area. So far, genome sizes, cell and nuclear volume were correlated. The karyotypes varied depending on the species, but without being directly associated with genome size.

Veronika Kapustová (Institute of Experimental Botany of the Academy of Sciences of the Czech Republic) built on the reports of apparent 18S-5.8S-26S rDNA relocation along the chromosomes of several grasses during their evolution [Dubcovsky and Dvorák, 1995]. To address this nomad-like behavior of rDNA loci in Triticeae grasses, she presented how new technologies including Bionano optical maps and $\mathrm{Hi}-\mathrm{C}$ data may help to define the exact positions and arrangements of rDNA loci. 
Antonia Simon (University of Vienna, Austria) presented how tandem repeats from 2 Capsicum species were used for the identification of all chromosome pairs. Comparative karyotyping revealed intraspecific polymorphism and allowed to trace genomic restructuring events during speciation and domestication.

Ludmila Cristina Oliveira (Biology Center Academy of Sciences of the Czech Republic) informed us about the genus Cuscuta, comprising species with mono- and holocentric chromosomes. She compared the centromeric histone $\mathrm{H} 3$ variant $\mathrm{CENH} 3$ among those species, finding large differences across the subgenera. Whereas species with monocentric chromosomes contained canonical active centromeres made up of satellite DNA and CRMtype chromoviruses [Neumann et al., 2011], some holocentric species were unusually depleted of CENH3. This raises the question if the CENH3 function even plays a role in Cuscuta holocentromeres [Oliveira et al., 2019].

Lastly, Thomas Schmidt's group (TU Dresden, Germany) presented much of the ongoing work on which Thomas had a direct impact:

Ludwig Mann and Kathrin Seibt showcased their new softwares for the analysis of extrachromosomal DNA as well as the FlexiDot software to generate visually attractive dotplots [Seibt et al., 2018].

Kathrin Seibt also presented her work on short interspersed nuclear elements (SINEs), more specifically the Angio-SINE superfamily [Seibt et al., 2019]. These usually diverse transposable elements harbor a surprisingly conserved $3^{\prime}$ module and occur in at least 46 plant genomes of 13 orders. SINEs with this domain are frequently enriched in and nearby genes, potentially impacting their expression or splicing.

Using the sugar beet (Beta vulgaris) genome, Beatrice Weber showed that tandem repeats and retrotransposons have more in common than previously thought. She presented sugar beet's uniquely mixed tandem repeat/retrotransposon landscape, with at least 8 retrotransposon families harboring different tandem repeats in their $3^{\prime}$ untranslated regions. To better understand whether the internal repeats invaded or emerged within the retrotransposons, she characterized 2 selected tandem repeat-retrotransposon partnerships.

How remnants of pararetroviral infections in the past may escape elimination was explained by Nicola Schmidt. Also working on the sugar beet genome, she identified 3 endogenous pararetrovirus (EPRV) families. Whereas 2 families still included intact copies, the third family was much more degenerated. Based on molecular and cytogenetic methods, she concluded that the EPRVs are em- bedded in highly compacted, repetitive regions, potentially providing safe havens preserving the EPRV elements.

Finally, Tony Heitkam traced the evolution of the allotetraploid crop quinoa (Chenopodium quinoa) starting from potential A- and B-genome precursor species [Heitkam et al., 2019]. For this, she used comparative wholegenome sequencing data in low coverage, Southern and in situ hybridization to identify and characterize the tandem repeat profiles of all 3 species. The diploid A- and B-genomes were marked by unequal satDNA amplification and subgenome-specific occurrence. She suggested scenarios for the evolution of the satellite DNAs before and after allopolyploidization and could exclude C. pallidicaule as quinoa's parental species.

\section{Outlook}

The Cytogenetics working group will meet again in 2021, with a venue and date yet to be specified (see www. facebook.com/GPZcytogenetic or https://gpz-online.de/ arbeitsgebiete/3-cytogenetik-chromosomenanalyse).

\section{Acknowledgement}

The conference organizers Tony Heitkam and Andreas Houben wish to thank the staff of TU Dresden's "Plant Cell and Molecular Biology" lab for help in all aspects of the meeting organization. Similarly, the IPK Gatersleben supported us on many levels, and pragmatically jumped in, if anything was unclear. Regarding this meeting report, T. Heitkam sincerely thanks Kathrin Seibt and Andreas Houben for critical proofreading of this manuscript.

\section{Disclosure Statement}

The author has no conflicts of interest to declare.

\section{Funding Sources}

We gratefully received financial support from the Society for Plant Breeding (GPZ), the KWS SAAT SE \& Co. KGaA, and the Technische Universität Dresden ("Flexibler Fördertopf Internationalisierung," grant number Flx1909_125). 


\section{References}

Báez M, Vaio M, Dreissig S, Schubert V, Houben A, Pedrosa-Harand A: Together but different: the subgenomes of the bimodal Eleutherine karyotypes are differentially organized. Front Plant Sci 10:1170 (2019).

Dubcovsky J, Dvorák J: Ribosomal RNA multigene loci: nomads of the Triticeae genomes. Genetics 140:1367-1377 (1995).

Heitkam T, Weber B, Walter I, Ost C, Schmidt T: Satellite DNA landscapes after allotetraploidisation of quinoa (Chenopodium quinoa) reveal unique $A$ and $B$ subgenomes. bioRxiv, DOI: 10.1101/774828 (2019).

Herklotz V, Kovařík A, Lunerová J, Lippitsch S, Groth M, Ritz CM: The fate of ribosomal RNA genes in spontaneous polyploid dogrose hybrids [Rosa L. sect. Caninae (DC.) Ser.] exhibiting non-symmetrical meiosis. Plant J 94: 77-90 (2018)

Hoang PTN, Schubert V, Meister A, Fuchs J, Schubert I: Variation in genome size, cell and nucleus volume, chromosome number and rDNA loci among duckweeds. Sci Rep 9:3234 (2019).

Ishii T, Schubert V, Khosravi S, Dreissig S, MetjeSprink J, et al: RNA-guided endonuclease - in situ labelling (RGEN-ISL): a fast CRISPR/ Cas9-based method to label genomic sequences in various species. New Phytol 222: 1652-1661 (2019).

Jiang J, Gill BS: Current status and the future of fluorescence in situ hybridization (FISH) in plant genome research. Genome 49:10571068 (2006).

Kopecký D, Horáková L, Duchoslav M, Doležel J: Selective elimination of parental chromatin from introgression cultivars of xFestulolium (Festuca $\times$ Lolium). Sustainability 11:3153 (2019).
Kuo YT, Chao YT, Chen WC, Shih MC, Chang SB: Segmental and tandem chromosome duplications led to divergent evolution of the chalcone synthase gene family in Phalaenopsis orchids. Ann Bot 123:69-77 (2019).

Milner SG, Jost M, Taketa S, Mazón ER, Himmelbach A, et al: Genebank genomics highlights the diversity of a global barley collection. Nat Genet 51:319-326 (2019).

Nemati Z, Harpke D, Kerndorff H, Gemicioglu A, Blattner FR: Saffron (Crocus sativus) is an autotriploid that evolved in Attica (Greece) from wild Crocus cartwrightianus. Mol Phylogenet Evol 136:14-20 (2019).

Nemeckova A, Wasch C, Schubert V, Ishii T, Hribova E, Houben A: CRISPR/Cas9-based RGEN-ISL allows the simultaneous and specific visualization of proteins, DNA repeats, and sites of DNA replication. Cytogenet Genome Res 159:48-53 (2019).

Neumann P, Navrátilová A, Koblížková A, Kejnovsky E, Hribova E, et al: Plant centromeric retrotransposons: a structural and cytogenetic perspective. Mob DNA 2:4 (2011).

Oliveira LC, Neumann P, Jang TS, Klemme S, Schubert V, et al: Mitotic spindle attachment to the holocentric chromosomes of Cuscuta europaea does not correlate with the distribution of CENH3 chromatin. Front Plant Sci, DOI: 10.3389/fpls.2019.01799 (2019).

Perničková K, Koláčková V, Lukaszewski AJ, Fan C, Vrána J, et al: Instability of alien chromosome introgressions in wheat associated with improper positioning in the nucleus. Int J Mol Sci 20:E1448 (2019).

Schmidt T, Heitkam T, Liedtke S, Schubert V, Menzel G: Adding color to a century-old enigma: multi-color chromosome identification unravels the autotriploid nature of saffron (Crocus sativus) as a hybrid of wild Crocus cartwrightianus cytotypes. New Phytol 222:1965-1980 (2019).
Schneider A: Untersuchungen über Plathelminthen, 1st ed (J. Ricker'sche Buchhandlung, Giessen 1873).

Seibt KM, Schmidt T, Heitkam T: FlexiDot: highly customizable, ambiguity-aware dotplots for visual sequence analyses. Bioinformatics 34:3575-3577 (2018).

Seibt KM, Schmidt T, Heitkam T: The conserved $3^{\prime}$ Angio-domain defines a superfamily of short interspersed nuclear elements (SINEs) in higher plants. Plant J, Epub ahead of print (2019).

Šimoníková D, Němečková A, Karafiátová $M$, Uwimana B, Swennen R, et al: Chromosome painting facilitates anchoring reference genome sequence to chromosomes in situ and integrated karyotyping in banana (Musa spp.). Front Plant Sci 10:1503 (2019).

Staňková H, Hastie AR, Chan S, Vrána J, Tulpová $\mathrm{Z}$, et al: BioNano genome mapping of individual chromosomes supports physical mapping and sequence assembly in complex plant genomes. Plant Biotechnol J 14:1523-1531 (2016).

Tulpová Z, Toegelová H, Lapitan NLV, Peairs FB, Macas J, et al: Accessing a Russian wheat aphid resistance gene in bread wheat by longread technologies. Plant Genome 12:180065 (2019).

Wassie AT, Zhao Y, Boyden ES: Expansion microscopy: principles and uses in biological research. Nat Methods 16:33-41 (2019).

Wu D, Ruban A, Fuchs J, Macas J, Novák P, et al: Nondisjunction and unequal spindle organization accompany the drive of Aegilops speltoides B chromosomes. New Phytol 223: 1340-1352 (2019).

Zsögön A, Čermák T, Naves ER, Notini MM, Edel $\mathrm{KH}$, et al: De novo domestication of wild tomato using genome editing. Nat Biotechnol 36:1211-1216 (2018). 